\title{
Características magnéticas y microestructurales de aceros eléctricos con alto silicio producidos por inmersión y recocido de difusión
}

\author{
T. Ros-Yañez, C. Alonso*,**, M. Gamarra, ${ }^{* * *}$, J. Barros* y Y. Houbaert \\ Resumen Los aceros eléctricos con alto contenido en silicio (hasta 6,5\% Si) son difíciles de procesar \\ usando procedimientos convencionales: fenómenos de ordenamiento hacen que el material \\ sea muy frágil para ser laminado en frío. El proceso de "inmersión en caliente y recocido de \\ difusión" es una vía alternativa para obtener aceros de alto contenido en silicio ( $\mathrm{Si}$ ) y, \\ eventualmente, también aluminio (Al), evitando los problemas durante la laminación. Los \\ substratos con hasta 3,5 m-\% de silicio se sumergen en un baño hipereutéctico de $\mathrm{Al}-\mathrm{Si}$ \\ para depositar en su superficie una capa rica en silicio. Un recocido posterior permite lograr \\ diferentes perfiles de concentración de este último como, por ejemplo, concentración \\ homogénea de 6,5\% en todo el espesor o perfiles específicos con por ejemplo 6,5\% de Si \\ en la superficie y $3,5 \%$ en el interior de la lámina. En estos materiales se obtuvieron \\ excelentes valores, en cuanto a pérdidas eléctricas.
}

Palabras clave Aceros eléctricos. Aceros con 6,5 \% Si. Recocido de difusión. Inmersión en caliente.

\section{Magnetic and microstructural characteristics of high-Si electrical steels produced by hot dipping and diffusion annealing}

\begin{abstract}
Electrical steels with high silicon content (up to $6.5 \mathrm{wt} \% \mathrm{Si}$ ) are difficult to process by conventional metallurgical routes: ordering phenomena make the material too brittle to be cold rolled. A hot dipping and diffusion annealing process appears to be an alternative production route to obtain steel with a high silicon and aluminium content avoiding rolling problems. Surface alloying with silicon $(\mathrm{Si})$ and aluminium $(\mathrm{Al})$ is achieved on a conventional steel substrate by hot dipping in a hypereutectic Al-Si-bath followed by a diffusion annealing treatment. The diffusion annealing allows the homogenization of the composition obtaining a homogeneous concentration of $6.5 \% \mathrm{Si}$ over the thickness depending on the temperature and annealing time necessary for the diffusion of the elements present in the coating. Also, these elements can be distributed in a controlled non-uniform way over the thickness of the sheet.
\end{abstract}

Keywords Electrical steels. $6.5 \%$ Si steels. Hot dipping. Diffusion annealing.

\section{INTRODUCCIÓN}

Las aleaciones $\mathrm{Fe}$-Si son muy usadas como materiales magnéticos blandos, especialmente en motores, generadores y transformadores. Aceros al silicio, hasta $3,5 \% \mathrm{Si}$, se utilizan ampliamente en estos equipos, pero las óptimas condiciones, en términos de pérdidas eléctricas y generación de ruido, no se alcanzan aún en ese rango de composiciones. Para aplicaciones eléctricas, las mejores propiedades se consiguen cuando se adiciona $6,5 \% \mathrm{Si}$ en el acero: la resistencia eléctrica aumenta y por consi- guiente disminuyen las pérdidas por Eddy-currents, mientras que la magnetoestricción se hace, prácticamente, cero. Sin embargo, es bien conocido que para contenidos en Si superiores a $4 \%$, las aleaciones $\mathrm{Fe}-\mathrm{Si}$ se hacen frágiles, siendo prácticamente imposibles de procesar por técnicas convencionales de laminación. La presencia de una superestructura ordenada del tipo $\mathrm{DO}_{3}$ (25\% atómico de $\mathrm{Si}$ ) es responsable de este comportamiento ${ }^{[1-3]}$.

El método de producción de acero de alto silicio por inmersión en un baño líquido de Al-Si, seguido por un recocido de difusión es un método

(*) Ghent University, Department of Metallurgy and Materials Science, Technologiepark 9, B-9052 Gent, Belgium.

$\left(*^{* *}\right) \quad$ University of Valladolid, Facultad de Ciencias, Paseo Prado de la Magdalena s/n, Valladolid, Spain. 
alternativo, desarrollado en el Departamento al que pertenecen los autores del presente trabajo, para la obtención de esta clase de acero, evitando los problemas en la laminación derivados de la fragilidad del material. Una chapa de acero de bajo contenido en silicio, todavía laminable hasta el espesor final deseado, se sumerge en un baño hipereutéctico de una aleación Al-Si. Al ser este baño hipereutéctico, durante la inmersión y formación de la capa de intermetálicos en la superficie del acero, se depositan cristales primarios de silicio puro. Durante el recocido de difusión, éste se difunde en la chapa de acero enriqueciéndola en Si hasta la composición deseada. Diferentes perfiles de concentración en silicio son posibles, como por ejemplo, concentración homogénea de $6,5 \%$ en todo el espesor o perfiles específicos con, por ejemplo, $6,5 \%$ de Si en la superficie y $3,5 \%$ de $\mathrm{Si}$ en el interior de la lámina ${ }^{[4-6]}$.

\section{MATERIALES Y PROCEDIMENTO EXPERIMEN- TAL}

Se seleccionaron 7 substratos para la inmersión con diferentes contenidos en silicio: 0,3, 1,3, 1,9, $2,4,3,2,3,3$ y $3,8 \%$ Si. De esta manera, se estudió el efecto del contenido inicial del silicio en la formación de las capas de intermetálicos durante la inmersión. Los experimentos se realizaron en un simulador comercial para el estudio de recubrimientos en continuo por inmersión en caliente ${ }^{[5]}$. Las muestras de dimensiones $200 \times 110 \mathrm{~mm}$ se precalentaron en el horno de infrarrojos del equipo hasta $850^{\circ} \mathrm{C}$, durante $60 \mathrm{~s}$ y bajo atmósfera reductora de $\mathrm{N}_{2}+5 \% \mathrm{H}_{2}$. Las inmersiones se realizaron en un baño $\mathrm{Al}-26 \% \mathrm{Si}$ a $800^{\circ} \mathrm{C}$, durante 5,20 ó $100 \mathrm{~s}$. Después de la inmersión las muestras se enfriaron rápidamente en nitrógeno ó lentamente $\left(20^{\circ} \mathrm{C} / \mathrm{min}\right)$ en el horno de infrarrojos. El recocido de difusión se llevó a cabo fuera del simulador, en tubos de cuarzo al vacío a temperaturas de 1.000 , 1.110 y $1.250^{\circ} \mathrm{C}$ durante $15,20,30$ y $40 \mathrm{~min}$.

\section{RESULTADOS}

\subsection{Características del recubrimiento deposita- do después de la inmersión}

Durante la inmersión del substrato en el baño se forman diferentes capas de compuestos $\mathrm{Fe}-\mathrm{Si}$ - $\mathrm{Al}$ en el recubrimiento. Para los tiempos de inmersión analizados aparecen siempre las mismas capas, modificándose su espesor en dependencia del tiempo

\section{Substrato}
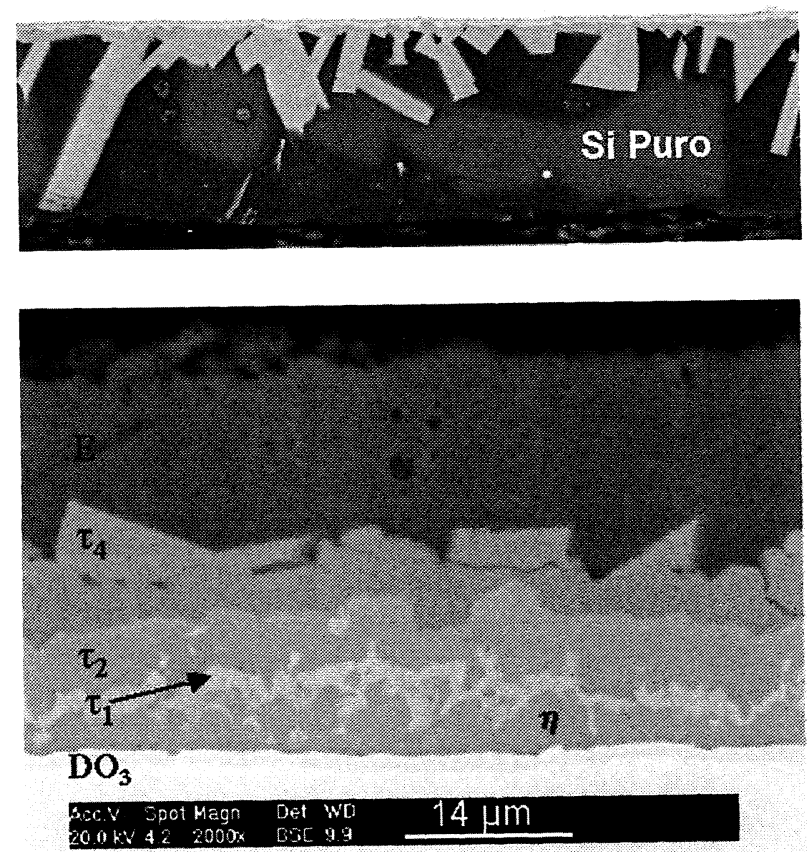

Figura 1. Apariencia de las capas depositadas después de la inmersión en el baño $\mathrm{Al}-\mathrm{Si}$ a $800^{\circ} \mathrm{C}$ : izquierda $5 \mathrm{~s}$ de inmersión, derecha con 20 s de inmersión.

Figure 1. Appearance of the deposited layers after the hot dipping in the Al-Si bath at $800{ }^{\circ} \mathrm{C}$ : left $5 \mathrm{~s}$ dipping time, right 20 s dipping time.

de inmersión ${ }^{[5]}$. La figura 1 muestra la microestructura típica del recubrimiento, identificándose las siguientes capas:

$$
\begin{array}{ccc}
\text { Fase ordenada } & \mathrm{DO}_{3}-\mathrm{Fe}_{3}(\mathrm{Al}+\mathrm{Si}) & \eta-\mathrm{Fe}_{2} \mathrm{Al}_{5} \\
\tau_{1}-\mathrm{Al}_{3} \mathrm{Fe}_{3} \mathrm{Si}_{2} & \\
\tau_{2}-\mathrm{Al}_{12} \mathrm{Fe}_{6} \mathrm{Si}_{5} & \text { F- Eutéctico } & \tau_{4}-\mathrm{Al}_{3} \mathrm{FeSi}_{2}
\end{array}
$$

En la capa eutéctica se han depositado cristales con $100 \%$ de Si (Fig. 1a), los cuales se observan en él recubriendo, en muestras inmersas hasta $5 \mathrm{~s}$ en el baño. Al incrementarse el tiempo de inmersión, el silicio puro que se depositó, se difunde hacia el substrato (Fig. 1b) y la capa de $\mathrm{DO}_{3}$ aumenta de espesor. Se observó una relación entre el crecimiento de esta capa y el contenido inicial de silicio en el substrato. En el gráfico de la figura 2 se observa cómo al aumentar el contenido inicial 


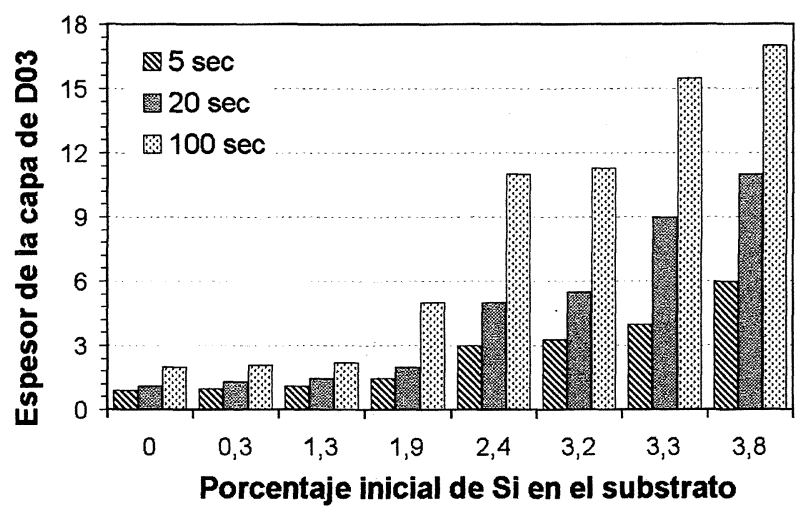

Figura 2. Variación del espesor de la capa $\mathrm{DO}_{3}$ en función del contenido inicial de $\mathrm{Si}$ en el substrato y para 3 tiempos de inmersión: 5,20 y $100 \mathrm{~s}$.

Figure 2. Evolution of the $\mathrm{DO}_{3}$ layer in function of the initial content of $\mathrm{Si}$ in the substrtae and for different dipping times: 5,20 and $100 \mathrm{~s}$.

de Si en éste, su difusión en el mismo es mayor, obteniéndose mayores espesores en la capa de $\mathrm{DO}_{3}$. La composición de Si y Al de esta capa ( $25 \%$ atómico de $\mathrm{Si}+\mathrm{Al}$ ) también depende del contenido inicial de $\mathrm{Si}$ en el substrato. Para substratos con contenidos en $\mathrm{Si}$ menores de 2,0 \% la composición es, mayoritariamente de $\mathrm{Al}$, mientras que para los de altos contenidos en $\mathrm{Si}$, prácticamente es una capa del tipo $\mathrm{Fe}_{3} \mathrm{Si}$ (Fig. 3).

\subsection{Recocido de difusión}

Primeramente, se estudió el efecto de la velocidad de calentamiento hasta la temperatura de recocido en la adherencia entre el recubrimiento y la mues-

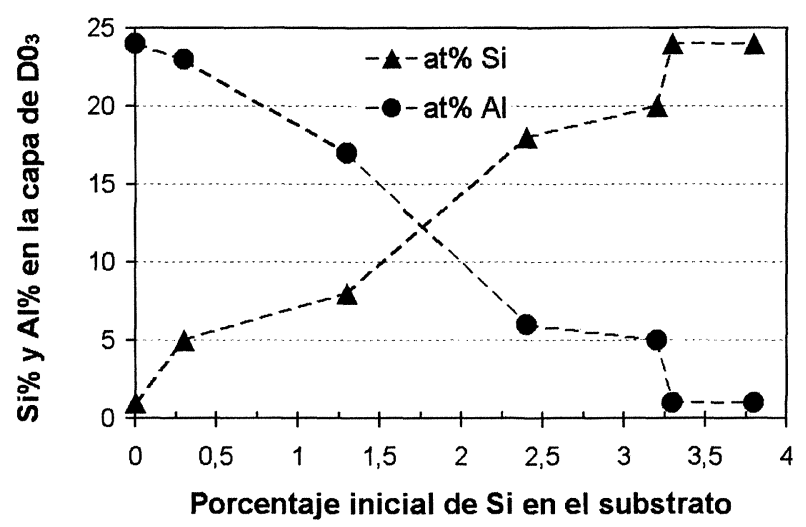

Figura 3. Evolución del porcentaje atómico de $\mathrm{Al}$ y $\mathrm{Si}$ en la capa $\mathrm{DO}_{3}$ en función del contenido inicial de $\mathrm{Si}$ en el substrato. Los datos corresponden a un tiempo de inmersión de $20 \mathrm{~s}$.

Figure 3. Concentration of $\mathrm{Al}$ at. \% and $\mathrm{Si}$ at. \% in the $\mathrm{DO}_{3}$ layer in function of the initial Si contents in the substrate. The data correspond to $20 \mathrm{~s}$ dipping time. tra. Velocidades de calentamiento superiores a $15^{\circ} \mathrm{C} / \mathrm{min}$ no se recomiendan en la literatura porque es posible el despegue de la capa debido a las diferencias en los coeficientes de dilatación térmica del substrato y del Si y/o sus intermetálicos ${ }^{[2 \text { y 3] }}$. Una serie de muestras se calentaron hasta $1.250^{\circ} \mathrm{C}$; una parte fueron introducidas directamente en el horno a la temperatura de tratamiento, lo que supone un calentamiento muy rápido (menos de 3 min para alcanzar la temperatura de recocido), mientras que el resto se calentaron a $10^{\circ} \mathrm{C} / \mathrm{min}$. En ningún caso, se observó el desprendimiento del recubrimiento y la difusión del Si dentro del substrato fue similar. Una vez que la capa $\mathrm{Fe}_{3}(\mathrm{Al}+\mathrm{Si})$ se forma durante la inmersión, la velocidad de calentamiento en el recocido de difusión no es crítica en términos de esfuerzo térmico, porque los coeficientes de expansión térmica de la capa de $\mathrm{DO}_{3}$ y el substrato son parecidos. Para el resto de los experimentos se utilizaron velocidades de calentamiento rápidas.

Durante el recocido de difusión el substrato se enriquece en $\mathrm{Si}$ y en Al: dependiendo de las temperaturas y de los tiempos utilizados es posible obtener diferentes perfiles de concentración. En la figura 4 se aprecian los perfiles de $\mathrm{Si}$ a lo largo del espesor en muestras de $0,35 \mathrm{~mm}$, para varias temperaturas de recocido. Es posible obtener una composición homogénea de $5 \%$ de $\mathrm{Si}$ en todo el espesor si las temperaturas de recocido son suficientemente altas ó un perfil específico de, por ejemplo, $4,5 \% \mathrm{Si}$ en la superficie, mientras que en el interior de la lámina se mantiene el contenido inicial

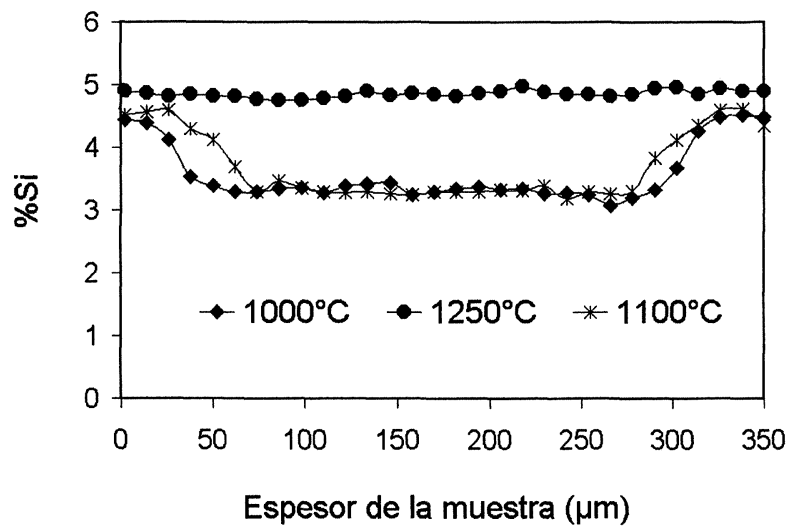

Figura 4. Perfiles de concentración de $\mathrm{Si}$ en muestras de $0,35 \mathrm{~mm}$ de espesor después de un recocido de difusión a diferentes temperaturas durante $30 \mathrm{~min}$.

Figure 4. Si concentration profiles of samples with $0.35 \mathrm{~mm}$ thickness after diffusion annealing for $30 \mathrm{~min}$ at different temperatures.

Rev. Metal. Madrid Vol. Extr. (2005) 222-226 
de Si (en este caso 3,2 \%). Ambas posibilidades son válidas en términos de pérdidas eléctricas, puesto que las corrientes de Eddy aparecen mas cerca de la superficie de la lámina que del centro y un gradiente en la concentración aumentará localmente la resistividad, modificando ventajosamente el flujo de estas corrientes y, por tanto, diminuyendo las pérdidas.

Si el recocido de difusión se lleva a cabo a $1.250^{\circ} \mathrm{C}$, dependiendo del tiempo de tratamiento se obtendrá, igualmente, un perfil de Si ó una concentración homogénea de éste en todo el espesor, si los tiempos son suficientemente largos (Fig. $5 \mathrm{a})$. La figura $5 \mathrm{~b}$ muestra las pérdidas eléctricas a $50 \mathrm{~Hz}-1$ tesla, en muestras recocidas a diferentes temperaturas: se aprecia cómo para las muestras recocidas a $1.250^{\circ} \mathrm{C}$ a partir de $20 \mathrm{~min}$, las pérdidas son de $0,8 \mathrm{~W} / \mathrm{kg}$. Sin embargo, como se observa en la figura 5a) y, aún con esos tiempos de recocido no se logra una distribución homogénea de Si en el espesor de la muestra, pero las pérdidas han alcanzado un valor mínimo y prácticamente constante.

En este trabajo, los valores mínimos de pérdidas eléctricas se obtuvieron para muestras cuyo substrato tenía inicialmente, antes de la inmersión, más de 3,2 \% Si y cuando se aplicaron tiempos de inmersión a partir de $20 \mathrm{~s}$. Estas muestras presentaron una capa de $\mathrm{DO}_{3}$, antes de aplicar el recocido de difusión, mucho mayor que el resto de los substratos con menor Si. La figura 6 muestra como se obtuvieron concentraciones homogéneas en el espesor de hasta $6,3 \% \mathrm{Si} \mathrm{y} \mathrm{4,5 \%} \mathrm{Al} \mathrm{cuando} \mathrm{se} \mathrm{apli-}$ có un recocido de difusión de $1.250^{\circ} \mathrm{C}$ durante 30 min, en muestras previamente inmersas durante $20 \mathrm{~s}$ en el baño Al-Si. Las pérdidas totales se redujeron a $50 \mathrm{~Hz}$ - 1 tesla, de $3,76 \mathrm{~W} / \mathrm{kg}$, en el substrato original, a $0,64 \mathrm{~W} / \mathrm{kg}$. A más altas frecuencias, $400 \mathrm{~Hz}-1$ tesla, las pérdidas totales se redujeron de $46,85 \mathrm{~W} / \mathrm{kg}$ a $10 \mathrm{~W} / \mathrm{kg}$.

\section{CONCLUSIONES}

El método de producción de acero de alto silicio por inmersión en un baño líquido de una aleación hipereutéctica $\mathrm{Al}-\mathrm{Si}$, seguido por un recocido de difusión, permite obtener aceros de alto contenido en Si y Al (hasta 6,3\% Si y 4,5\% Al). Los substratos de bajo contenido en silicio, se sumergen en el baño y durante la inmersión se forma un recubrimiento con capas de intermetálicos en la superficie del acero, donde se depositan cristales primarios de silicio puro. Durante el recocido de
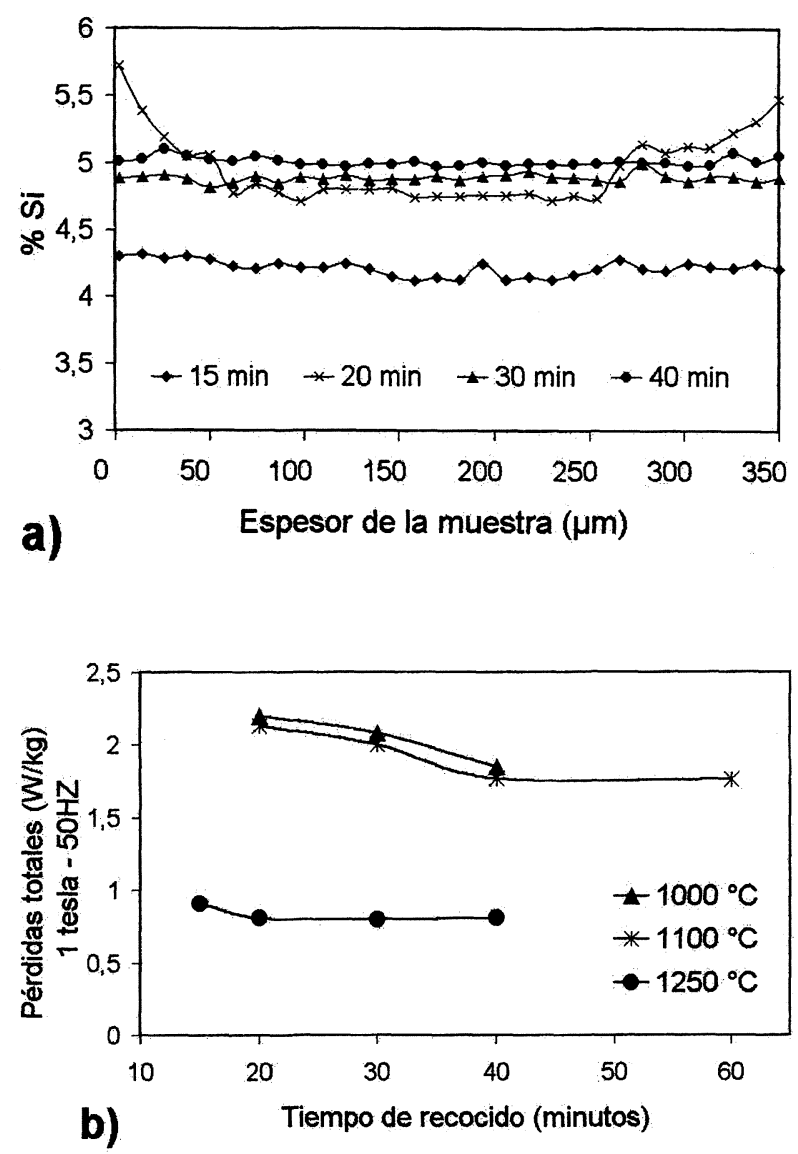

Figura 5. a) Perfiles de concentración de Si en función del tiempo de recocido para muestras tratadas a $1.250{ }^{\circ} \mathrm{C}$ (0,35 mm de espesor). b) Pérdidas eléctricas a 1 tesla, 50 $\mathrm{Hz}$ en función del tiempo de recocido para diferentes temperaturas (muestras con $0,35 \mathrm{~mm}$ de espesor).

Figure 5. a) Evolution of the Si concentration profiles with the annealing time at $1,250{ }^{\circ} \mathrm{C}$ in samples with $0.35 \mathrm{~mm}$ thickness. b) Iron loss at $1 \mathrm{~T}$ in function of the annealing time for different temperatures (samples with $0.35 \mathrm{~mm}$ thickness).

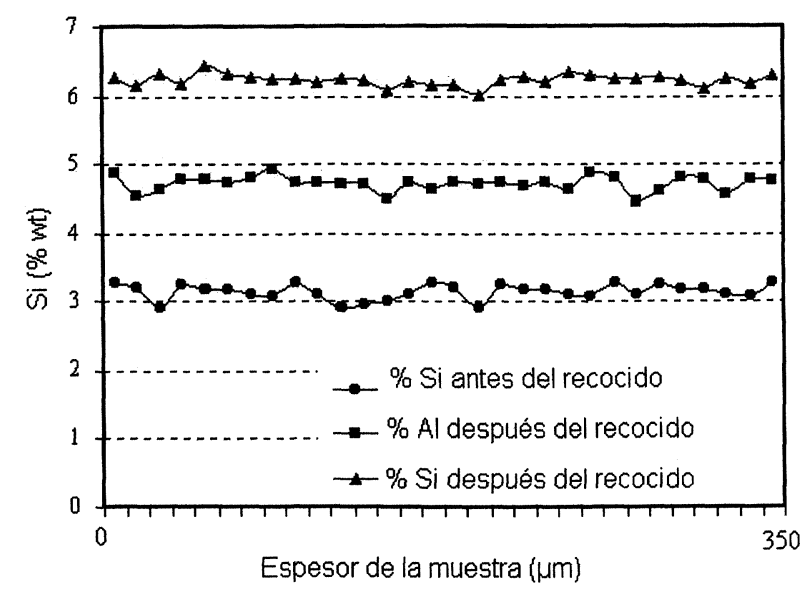

Figura 6. Perfiles de concentración $\mathrm{Al}$ y Si antes y después de recocido de difusión a $1.250{ }^{\circ} \mathrm{C}$ durante $30 \mathrm{~min}$.

Figure 6. Si and Al concentration profiles before and after the diffusion annealing at $1,250^{\circ} \mathrm{C}$ during $30 \mathrm{~min}$. 
Características magnéticas y microestructurales de aceros eléctricos con alto silicio producidos por inmersión y recocido de difusión T. ROS-YANEz, C. Alonso, M. GamarRa, J. BARROS Y Y. HOUBAERT

difusión, éste se difunde en la chapa de acero, enriqueciéndola en Si hasta la composición deseada. Diferentes perfiles de concentración en silicio son posibles, como concentraciones homogéneas de $6,5 \%$ en todo el espesor o perfiles específicos con, por ejemplo, 4,5\% Si en la superficie y 3,2 \% Si en el interior de la lámina. Se obtuvieron excelentes valores de pérdidas eléctricas: $0,64 \mathrm{~W} / \mathrm{kg}$ a 50 $\mathrm{Hz}-1$ tesla y $10 \mathrm{~W} / \mathrm{kg}$ a $400 \mathrm{~Hz}-1$ tesla.

\section{REFERENCIAS}

[1] A.J. MOSES, IEEE 137 (1990) 233-245.

[2] A.J. Moses y G.J. Thursby, J. Mater. Sci. 18 (1983) 1.657 1.665 .

[3] J. Molina, C. Garcia y J. Gil, Scri. Mater. 41 (1999) 729. 735.

[4] T. Ros-Yañez, Y. Houbaert y V. Gomez, J. Appl. Phys. 91 (2002) 7.857-7.859.

[5] T. Ros-Yañez e Y. Houbaert et al., Rev. Metal. Madrid 36 (2000) 339-347.

[6] Y. Houbaert y T. Ros-Yañez, European, Patent, UK 011703.5, US 60/299, 056 (2002). 